\title{
STATUS OF THE MAX-LAB INJECTOR PROJECT
}

\author{
B. Anderberg, Å. Andersson, M. Demirkan, M. Eriksson M. Georgsson, G. LeBlanc, L-J Lindgren, \\ L. Malmgren and S. Werin, MAX-lab, Lund, Sweden
}

\begin{abstract}
The status of the new injector system for MAX-lab is presented. The electron source is a thermionic RF-gun that has been designed at MAX-lab. The new injector will consist of two linac structures equipped with SLED [1] cavities. This will produce an electron beam with an energy of $250 \mathrm{MeV}$. This beam will be recirculated in order to obtain a $500 \mathrm{MeV}$ beam.
\end{abstract}

\section{INTRODUCTION}

The MAX-lab national laboratory is currently replacing its old $100 \mathrm{MeV}$ racetrack microtron with a new injector system for the storage rings. The injector will produce electrons for injection at $250 \mathrm{MeV}$ into the MAX I storage ring, and $500 \mathrm{MeV}$ into the storage rings MAX II and MAX III. The MAX I storage ring is also operated as a pulse stretcher and the energy for this mode will be raised from $100 \mathrm{MeV}$ to $250 \mathrm{MeV}$. The source should also be able to provide electrons for possible installations of FELs in the Infrared and/or VUV in the future.

The basic principle of the new injector is to create a compact machine at a moderate cost. The injector should be able to operate in several different modes (Table 1).

Despite a long tradition of racetrack microtrons at MAX-lab, a linac solution has been chosen. Two features have been added to the traditional linac solution. First, the linacs are equipped with SLED cavities. These allow an increase of roughly $80 \%$ of the extracted energy. Second, the electron beam is recirculated once through the linacs. In this way a $15 \mathrm{~m}$ section with two $75 \mathrm{MeV}$ linacs can be boosted to a final energy of $500 \mathrm{MeV}$.

The complete new injector is designed to fit into the existing microtron cave, directly underneath the MAX I storage ring (see Fig. 1).

Some additional radiation shielding will be added to ensure safe conditions even with a more powerful source. The two linac structures will hang from the ceiling and the recirculation line will follow close to the floor level. The overall length of the system is $15 \mathrm{~m}$.

Table 1 Operation modes

\begin{tabular}{|c|c|l|}
\hline Energy & Mode & \\
\hline $100-125$ & SLED & Inject MAX I and MAX II (stage 1) \\
\hline 250 & SLED & $\begin{array}{l}\text { Injector for MAX I, SR and pulse } \\
\text { stretcher. (stage 2) }\end{array}$ \\
\hline 500 & SLED & $\begin{array}{l}\text { Injector for MAX II and the new ring. } \\
\text { (stage 3) }\end{array}$ \\
\hline $30-75$ & Classical & Driver for IR-FEL. \\
\hline $200-500$ & SLED & UV-FEL. \\
\hline
\end{tabular}

\section{THE GUN STRUCTURE}

\subsection{General Layout}

The basic idea behind the design is a gun with no need for a buncher cavity or a pre-accelerator linac. The choice has fallen on an RF-gun. A thermionic cathode was chosen for simple operation.

When choosing a thermionic cathode, back bombardment has to be considered. The gun is not designed to operate at extreme bunch charges (currents) and the demands for emittance are not too demanding. In

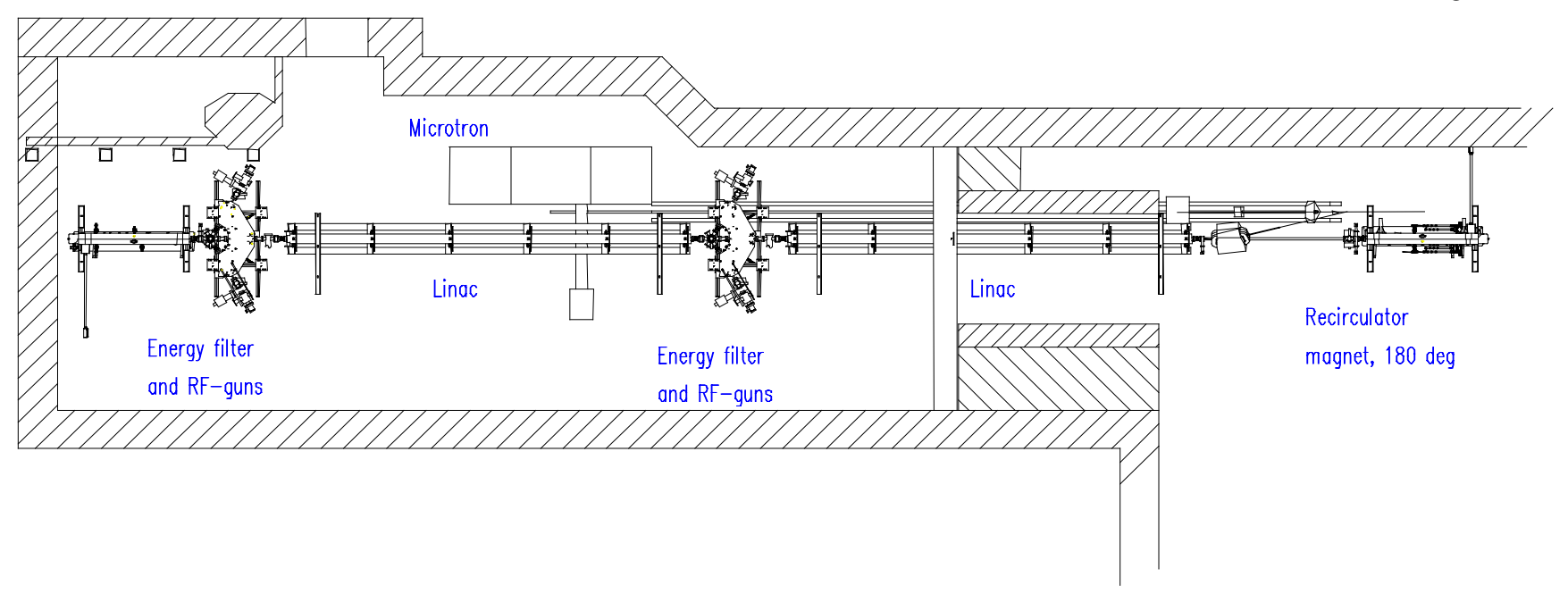

Figure 1: Layout of the recirculator. 
addition, the energy spread will be defined by the linac system as a direct consequence of the electron bunch length from the gun.

Several gun designers [2,3 e.g.] have addressed the problem of back bombardment. A common solution has been to introduce an extra "zero field" cavity to elongate the gun and to operate the excitation of the gun such that the first cavity has around $40 \%$ of the field amplitude of the main accelerating cavity. Thus a design with $1 / 2+1 / 2+1$ cell has been chosen with a field relation of 2.6 operating in $\pi / 2$ mode. This solution is similar to [3].

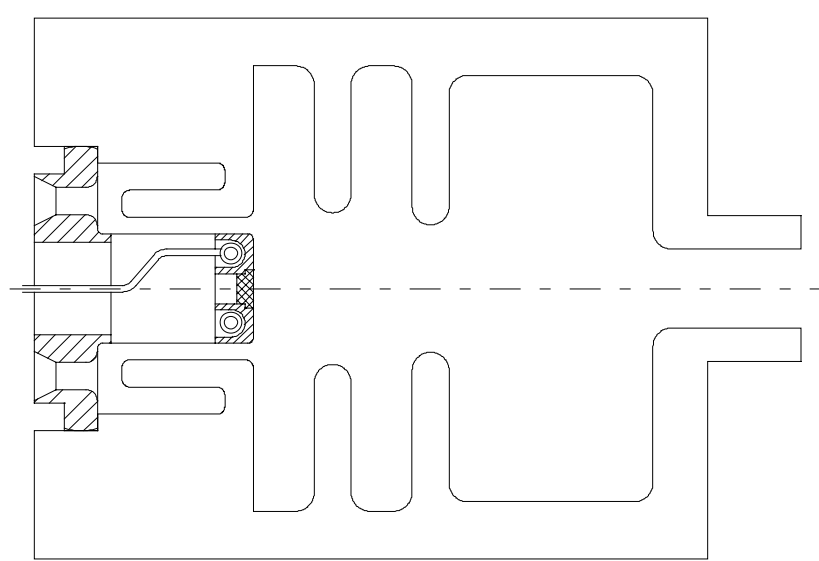

Figure 2: Layout of gun, RF-choke and cathode.

\subsection{Cathode}

Since cathode is heated to around $1000^{\circ} \mathrm{C}$, a proper insulation has to be made between it and the gun structure. At the same time a proper electrical contact has to be assured. Here the cathode is mounted via an RFchoke ( $\lambda / 2$ termination) (see Fig. 2). The choke is terminated in the end and the $\lambda / 2$ "moves" the termination to the entrance hole of the choke.

\subsection{Q-value measurements}

The Q-value was determined by transmission measurements between two E-field probes. The positions of the probes were adjusted for minimum coupling to the cavity. The wave-guide was terminated with a short circuit at a distance of 5 times $\lambda / 4$ which gives an open circuit at the coupling hole. This minimises the loading from the wave-guide. The achieved $\mathrm{Q}_{0}$-value was 12000 . Simulations gave an unloaded Q-value of 15000. This value was not expected to be reached due to additional losses in the wave guide system that are not considered in the simulation. The coupling factor $\beta$ was measured to be about 2 .

\subsection{Measurements with RF power}

The pressure in the gun was $3 * 10^{-7}$ mbar with the temperature controlling system running at $40^{\circ} \mathrm{C}$ before the $\mathrm{RF}$ power was switched on. The gun was tuned by measuring the reflected power while the temperature was changed. At a temperature of $47^{\circ} \mathrm{C}$ the gun was at resonance, which corresponds to the lowest value of reflected power. The conditioning of the gun was done starting from a low power level where it was running until the arcing stopped and then the power was increased to the next level. This process was done in several steps until it was possible to reach $2 \mathrm{MW}$ RF power in the gun. The power was measured by means of a coupling loop in one of the cavities The pressure in the gun increased to $1.3 * 10^{-7}$ mbar during the RF measurements.

\section{ENERGY FILTER}

An RF-gun with a thermionic cathode suffers from having a long low energy tail in the electron bunch. An energy filter has been designed to remove this tail and to provide opportunities to control the bunch length. The filter consists of two $60^{\circ}$ bending magnets and 5 quadrupole magnets as seen in Fig. 3. The dispersion allows filtering of the low energy electrons by a slit between the two bending magnets.

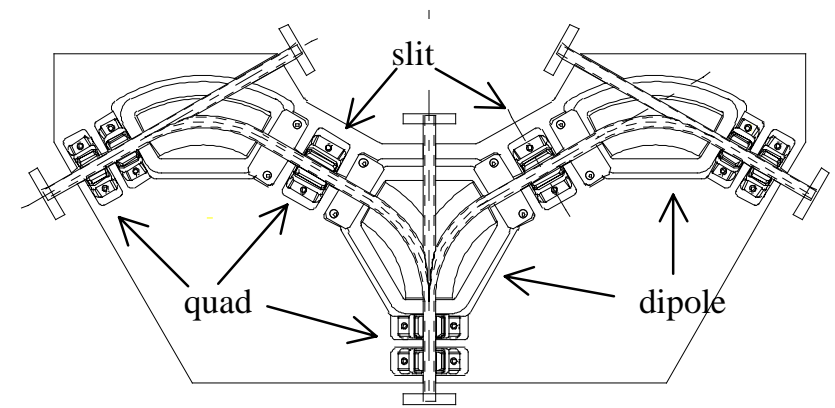

Figure 3. The energy filter. Entrance from gun at left and right. Exit to linac at middle bottom.

\section{THE LINAC SYSTEM}

The two linac sections are produced by Accel, tuned at DESY and already delivered to MAX-lab. These structures are identical to the ones delivered to the 100 $\mathrm{MeV}$ injector for SLS (PSI, Switzerland). In a standard configuration using one $35 \mathrm{MW}$ klystron per linac an electron energy of $75 \mathrm{MeV}$ can be achieved. In the MAXlab set up, a system with SLED cavities boosts this energy to above $125 \mathrm{MeV}$. The parameters of the linacs are shown in Table 2.

Table 2. The LINAC system

\begin{tabular}{|l|l|l|}
\hline Type & TWT & \\
\hline Length & 5.2 & $\mathrm{~m}$ \\
\hline Q & 10.000 & \\
\hline Frequency & 3 & $\mathrm{GHz}$ \\
\hline Klystron power & 35 & $\mathrm{MW}$ \\
\hline Energy gain (no SLED, 0 A) & 77.35 & $\mathrm{MeV}$ \\
\hline Energy gain (SLED, 0 A) & 138.7 & $\mathrm{MeV}$ \\
\hline Energy gain (SLED, 50 mA) & 136.5 & $\mathrm{MeV}$ \\
\hline Peak gradient increase w/SLED & x 2.63 & \\
\hline $\begin{array}{l}\text { Peak integrated gradient } \\
\text { increase w/ SLED }\end{array}$ & $\mathrm{x} 1.793$ & \\
\hline
\end{tabular}




\subsection{SLED system}

The idea of energy doubling uses storage cavities in which energy is accumulated and rapidly released to the linacs. There is of course a cost for this energy boost. The pulse to the linacs is considerably shorter than in the ordinary case. By recirculation, the extracted electron pulse will be half of the linac pulse at most. In our case we will extract a $100 \mathrm{~ns}$ pulse at $500 \mathrm{MeV}$. As our storage rings are 100, 100 and $300 \mathrm{~ns}$ each this is a very convenient number.

The SLED cavities are being manufactured and some initial measurements have been made. The Q-value before the final cut was 86000 . We now expect about 96000 in Q-value.

The cavity-coupling factor $\beta$ was measured to be 5.3. The resonance frequency after the final cut is 2997,6 $\mathrm{MHz}$ at room temperature. It should be $2998 \mathrm{MHz}$ but could easily be adjusted using a tuning screw in the bottom of the cavity. The $3 \mathrm{~dB}$ hybrids are brazed and ready for test measurements.

\section{$4.2 R F$-system}

Two new 35 MW klystrons to supply the linacs are installed and tested. These were conveniently placed in an adjacent room next to the existing $3 \mathrm{GHz}$ klystron.

\section{THE RECIRCULATOR}

The recirculator recycles the electron beam after the first transit of the linacs, back to the first linac for the second transit under isochronous conditions. The system is designed to work at $250 \mathrm{MeV}$.

Each $180^{\circ}$ bend should be close to a drift section matrix. These are also achromats to first and second order of energy. The $180^{\circ}$ bend consists of three bending magnets and five quadrupole magnets. The first order momentum compaction is adjusted to zero and the contribution from energy errors to second order for the length co-ordinate are adjusted to zero.

\subsection{Magnets}

The magnets of the recirculator are machined out of two solid plates of $70 \mathrm{~mm}$ iron (Fig. 4). The two plates are arranged such that they form a "door". The coils and the vacuum chamber are mounted for the magnets and the "door closed". The whole $180^{\circ}$ bend thus forms one package.

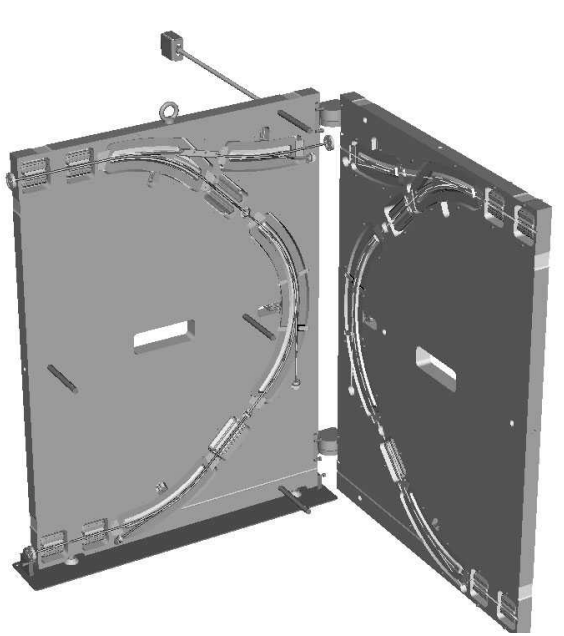

Figure 4. The recirculator magnets are machined out of one solid iron plate.

\subsection{Chicane}

The circumference of the recirculator must be precisely a multiple of the linac wavelength $99.974 \mathrm{~mm}$ (frequency $2.9987 \mathrm{GHz}$ ). Therefore the circumference is chosen to be $1 \mathrm{~mm}$ shorter than such a multiple and a chicane is added in the recirculator return path. This chicane can add up to $2 \mathrm{~mm}$ to the length. It consists of three magnets with a spacing of $1.0 \mathrm{~m}$. The beam in the central magnet is displaced $45 \mathrm{~mm}$ from the straight beam at maximum deflection.

\section{SUMMARY}

A cost effective and compact solution for a $500 \mathrm{MeV}$ linac based injector has been developed. The performance is expected to fulfil the current needs at MAX-lab. The machines also provide excellent opportunities for new developments. The first linac and the energy filter have been installed. The electron gun has been conditioned with RF power and tuned with a temperature control system. The two 35 MW klystrons have been installed and tested. The recirculator has been assembled and the SLED cavities are under construction.

\section{REFERENCES}

[1] Z. D. Farkas, SLAC-PUB-1453 (1974)

[2] V.V. Mitrochenko, PAC 97

[3] E. Tanabe et al. LINAC98

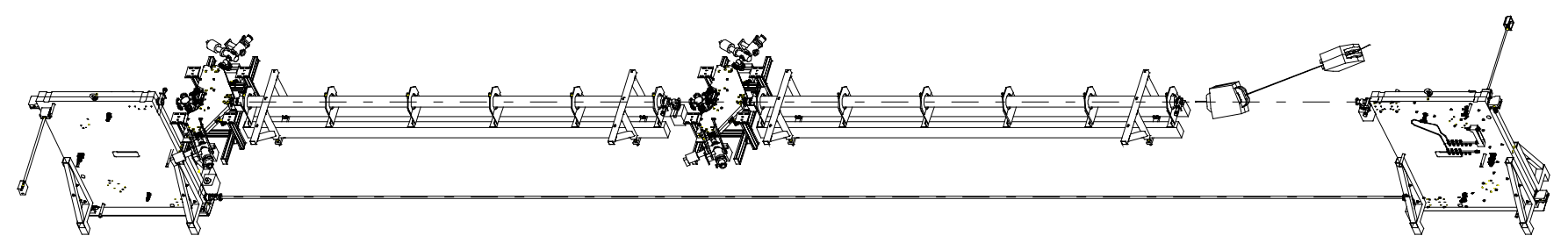

Figure 5. The injector system 